\title{
EDUCACIÓN EN SALUD: EN LA BÚSQUEDA DE METODOLOGÍAS INNOVADORAS
}

\author{
HEALTH EDUCATION: IN SEARCH OF \\ INNOVATIVE METHODOLOGIES
}

\author{
Silvia Barrios Araya* \\ M. Patricia Masalán Apip ${ }^{* *}$ \\ María PAZ COOK ${ }^{* * *}$
}

\begin{abstract}
RESUMEN
La educación en salud es un proceso complejo que requiere de la incorporación de conocimientos, habilidades, actitudes y valores necesarios para formar profesionales en el área, además de estrategias que permitan una evaluación integral de las competencias adquiridas. El objetivo de este trabajo es determinar el estado del arte existente acerca del uso de metodologías innovadoras en aprendizaje y evaluación para el desarrollo de habilidades clínicas en el área de la salud. La literatura revisada destaca metodologías tales como Examen Clínico Objetivo Estructurado, Instrucción y aprendizaje con ayuda computacional, Programas de aprendizaje basados en Internet, Programas basados en computación Multimedia, y Simulación de realidad virtual, cuyo enfoque educativo permite a los estudiantes de enfermería aplicar e integrar los conocimientos recién adquiridos con la experiencia clínica, de manera de generar mayor seguridad y confianza previo a la llegada al paciente verdadero. Estos métodos favorecen la autoconfianza en los estudiantes al ser tecnologías que propician el aprendizaje en un entorno seguro, provocando altos niveles de rendimiento clínico, académico y en la satisfacción con el curso. Además, estas tecnologías permiten el desarrollo de estrategias cognoscitivas superiores, más que la adquisición de solo conocimiento, lo que a la larga fomenta el logro de competencias. La implementación de tecnologías de este tipo requiere del fortalecimiento de los equipos de trabajo para la planificación e implementación de cambios. Sin embargo, las experiencias de simulación en un laboratorio nunca reemplazarán las experiencias clínicas con pacientes verdaderos.
\end{abstract}

Palabras clave: Educación, enfermería, metodologías, salud.

\begin{abstract}
Health education is a complex process that requires the incorporation of knowledge, skills, attitudes and values required to train professionals in the area, as well as strategies for a comprehensive assessment of the skills acquired. The aim of this review is to determine the existing state of the art regarding the use of innovative methodologies in learning and development evaluation of clinical skills in the area of health. The literature review include methodologies such as Objective Structured Clinical Examination, Instruction and Computer-assisted learning, Learning programs based on Internet, Multimedia computer-based programs and Virtual reality simulation, whose approach to education allows students to apply and integrate knowledge newly acquired and clinical experience, in order to generate greater security and confidence prior to actual patient arrival. These methods encourage self-confidence in the nursing students to be conducive to learning technologies in a secure environment, leading to high clinical performance, academic and satisfaction with the course. Another important point is that these technologies enable the development of cognitive strategies rather
\end{abstract}

\footnotetext{
*Enfermera. Docente Pontificia Universidad Católica, Escuela de Enfermería, Santiago - Chile. Email: sbarrios@uc.cl

** Enfermera Matrona, MSP, Pontificia Universidad Católica, Escuela de Enfermería, Santiago - Chile. Email: mmasalan@uc.cl

${ }^{* * *}$ Enfermera, MCE@), MEpi®, Pontificia Universidad Católica, Departamento de Salud Pública, Santiago - Chile. Email: mpcook@uc.cl
} 
than acquiring knowledge, which ultimately promotes skills. The implementation of such technologies requires a stronger work teams for planning and implementing change. However, simulation experiments in a lab will never replace clinical experiences with actual patients.

Key words: Education, nursing, methodologies, health.

Fecha recepción: 01/06/2010 Fecha aceptación: 20/12/2010

\section{INTRODUCCIÓN}

El aprendizaje en adultos es un proceso complejo compuesto por una serie de factores esenciales para su concreción. Para que este aprendizaje sea efectivo se deberían contemplar al menos tres áreas: cognitiva, afectiva y psicomotora, las que son fundamentales en la formación de los estudiantes para brindar calidad en el cuidado de la salud.

El plano cognitivo considera cinco niveles referentes a procesos mentales identificables como, recuerdo, comprensión, análisis, síntesis y aplicación. Las dos primeras corresponden a niveles básicos para que se den cualquiera de los niveles subsiguientes (1). Considerar estos factores en el proceso educativo permite entregar contenidos en forma coherente, precisa, sencilla y que estimulan conductas críticas frente a un problema (2).

Por otro lado, la educación en salud agrega variables que hacen al proceso educativo aun más complejo ya que requiere de la incorporación de conocimientos, habilidades, actitudes y valores necesarios para formar futuros profesionales en el área. Asimismo este proceso educativo debe ser alineado con el contexto, los rápidos cambios socioculturales y las necesidades emergentes (3).

De lo anterior se desprende que no sólo se requiere de un proceso educativo adecuado para el desarrollo de las competencias en el área de la salud, sino además estrategias que permitan una evaluación integral de las competencias adquiridas. Sin embargo, tradicionalmente, la "evaluación se ha basado en la medición de conocimientos mediante la aplicación de diversos tipos de exámenes” (4), que, según Triviño et al., no siempre cumplen con los criterios de objetividad, validez y confiabilidad (5). Esta situación ha llevado a la necesidad imperiosa de desarrollar nuevos métodos de educación y evaluación, destinados a evaluar competencias clínicas.

En la literatura internacional se encuentra una variedad de concepciones de lo que es una competencia profesional; es claro que desde el punto de vista puramente lingüístico el Diccionario de la Real Academia Española de la Lengua la describe como pericia, aptitud, idoneidad o incumbencia para hacer algo o intervenir en un asunto determinado (6). En cambio otros autores mencionan que "las competencias profesionales son las que permiten al individuo solucionar los problemas inherentes al objeto de su profesión en un contexto laboral específico, en correspondencia con las funciones, tareas y cualidades profesionales que responden a las demandas del desarrollo social" (7).

Asimismo, Ortiz (8) hace énfasis en que las competencias requieren de una integración de conocimientos científicos, habilidades y capacidades que se relacionan con el ejercicio de una profesión. Además considera las motivaciones, sentimientos, necesidades y valores relacionados que hacen que el desempeño del profesional sea eficaz y eficiente dentro del contexto social en el cual se desarrollará.

Por otro lado, en el área de la salud es necesario considerar el término competencia clínica como "el conjunto de atribuciones mul- 
tidimensionales que incluyen conocimientos teóricos, habilidades clínicas, relaciones interpersonales, solución de problemas, juicio clínico y destrezas técnicas" (4), por lo que ésta debe ser evaluada considerando las tres áreas psicoeducativas previamente descritas, cognitiva, afectiva y psicomotora.

De todo lo anteriormente expuesto se desprende, por un lado, el contar con metodologías que permitan proveer un aprendizaje efectivo y, por otro, contar con sistemas de evaluación adecuados para este enfoque. Dentro de ellos destacan metodologías tales como Examen Clínico Objetivo Estructurado (ECOE), Instrucción con ayuda computacional (CAI), o Aprendizaje con ayuda computacional (CAL), Programas de aprendizaje basados en Internet, Programas basados en computación Multimedia y Simulación de realidad virtual, los cuales serán detallados más adelante.

Este trabajo nace de la motivación de docentes de la Escuela de Enfermería de la Pontificia Universidad Católica de Chile, quienes en su afán de mejorar la calidad de la docencia buscan encontrar métodos educativos y evaluativos acordes a las necesidades actuales. Su objetivo es determinar el estado del arte existente acerca del uso de metodologías innovadoras en aprendizaje y evaluación para el desarrollo de habilidades clínicas en el área de la salud.

\section{MATERIAL Y MÉTODO}

Durante el periodo de agosto 2006 a julio 2009 se revisaron 51 artículos, extraídos de las bases de datos PubMed y ProQuest, así como de las revistas de origen, cuyos descriptores fueron metodologías, enfermería y educación. Se seleccionaron 39, cuyos años de publicación fluctúan entre 1997 y 2009. Posterior a esto se agruparon los estudios en cuatro grupos de acuerdo a las metodologías planteadas anteriormente.

\section{RESULTADOS Y DISCUSIÓN}

En esta sección se presentarán los resultados obtenidos en la búsqueda de literatura respecto a las principales metodologías innovadoras que se están utilizando a lo largo del mundo.

\section{Examen Clínico Objetivo Estructurado (ECOE)}

Dentro de la labor docente es importante utilizar un instrumento que permita establecer en los distintos momentos del proceso la calidad con que se van cumpliendo los objetivos de las diferentes asignaturas. Gracias a los avances de la ciencia y las modificaciones curriculares es que se han implementado nuevas metodologías de enseñanza y sistemas de evaluación (9).

De acuerdo a Triviño et al., el valor de cambiar la evaluación está en reflejar la importancia de aprender lo que se necesita saber y no sólo lo que los estudiantes saben que será evaluado (5). De este modo Harden et al. diseñan y aplican un Examen Clínico Objetivo Estructurado (ECOE), destinado a evaluar competencias clínicas (10).

Según Hassel, el ECOE "generalmente consiste en un circuito de estaciones" que, actualmente está siendo "utilizado por un gran número de instituciones de salud, escuelas de salud y sociedades científicas" (11).

"En cada estación, el educando es enfrentado a una pregunta específica, tal como aspectos de la historia o examinación del paciente", y es evaluado de acuerdo a una pauta por parte de un examinador. "Después del tiempo establecido para la pregunta, típicamente 5-15 minutos, suena un timbre y el candidato debe avanzar hacia otra estación del circuito" (11).

La selección de las situaciones clínicas se realiza habitualmente según la prevalencia, gravedad clínica, importancia de la prevención y del diagnostico precoz, complejidad 
del caso y evaluación de la capacidad resolutiva (12).

Pedregal et al. y Triviño et al. explican que las situaciones clínicas se realizan con pacientes estandarizados, fantomas, interpretación de imágenes clínicas, preguntas abiertas y selección múltiple, que pueden incluir grabaciones de video, fotografías, radiografías, curvas de evaluación nutricional, exámenes del laboratorio, entre otros elementos $(5,12)$.

Para calificar, se utilizan listas de comprobación de cada ítem, determinadas por los examinadores o los pacientes estandarizados (en inglés SPs) de la facultad. El uso de pacientes reales y simulados como SPs en el ECOE crea un ajuste de la vida real para el estudiante o residente (13).

El ECOE permite que el mismo problema clínico sea presentado a muchos examinados, en un ajuste altamente estructurado, en el cual los criterios objetivos se puedan utilizar para evaluar a cada participante directamente (13). Este examen proporciona una ventaja única en la capacidad clínica de estudiantes o de residentes (5).

Según Toledo et al., los resultados obtenidos permiten evaluar la competencia clínica a través del ECOE en dos perspectivas: las calificaciones obtenidas que reflejan el rendimiento académico y las ventajas de aplicar un examen que integra la evaluación de las áreas cognoscitivas con las habilidades clínicas e interpersonales (4).

En cuanto al rendimiento, Kurz et al. coinciden con Jiménez et al, en que los resultados del ECOE son superiores al índice académico y en el nivel de satisfacción con el curso. Jiménez et al. explican que esto podría producirse debido a "que existe una profundización en el estudio por parte de los alumnos, al enfrentarse a un método evaluativo diferente y que, además, motiva la autopreparación de los docentes" $(9,14)$.

Además, este autor explica que en el promedio ponderado acumulado se consideran otras asignaturas que pudieran tener resul- tados deficientes, lo que debiera motivar a un análisis de la situación y al perfeccionamiento del plan de estudios. Resulta interesante observar que en el mismo estudio las malas calificaciones disminuyen al igual que las excelentes, aumentando las calificaciones buenas e intermedias. Es decir, el ECOE eleva estas notas, ya que "además de la profundización en el estudio, existen condiciones objetivas en el acto evaluativo, al garantizarse igualdad de exigencias y posibilidades para todos los estudiantes" y que la disminución de las notas excelentes puede deberse a una mayor exigencia.

Triviño et al. coinciden en que el ECOE es un método exitoso pues es un procedimiento adecuado para evaluar a una gran cantidad de estudiantes simultáneamente y permite la medición de objetivos propuestos en todos los dominios e involucra una amplia gama de dominios clínicos presentes en los programas de estudio (5). Además, permite estandarizar criterios para homogeneizar una evaluación a nivel internacional con respecto a la evaluación de los alumnos en el pregrado y puede ser implementado como complemento de otros instrumentos de evaluación utilizados tradicionalmente.

Toledo et al. concluyen que este instrumento permitió la integración de las 3 dimensiones de la evaluación educativa y una evaluación más objetiva de los atributos multidimensionales que constituyen la competencia clínica (4).

Con este método, "no sólo se contribuye a un análisis de la teoría de evaluación, sino que es posible determinar el grado de cumplimiento de las exigencias en la formación de los nuevos profesionales para la salud, y con ello la competencia de éstos para asumir sus responsabilidades" (9).

Sin embargo, este no es un método exento de problemas, especialmente en un primer momento. Por ello, Triviño et al. recomiendan el fortalecimiento de los equipos de trabajo para la planificación e implementación 
de cambios modernizadores en la docencia de pregrado, ya que el uso de SPs y ECOE requieren de más horas de preparación de los docentes que los métodos tradicionales de aprendizaje, mensaje que coincide con un estudio realizado en el año 2009 por Kurz et al. $(5,14)$.

En el mismo tenor, Hassell et al. plantean como una desventaja que la evaluación de actividades de mayor complejidad como la comunicación del paciente y el planteamiento de diagnósticos sean evaluados a través de este tipo de modelo y que por lo mismo no se debe olvidar la importancia del tutor clínico dentro del proceso de aprendizaje (11). También, Pedregal et al. aportan con otra desventaja, que este procedimiento puede causar estrés a los participantes (12).

\section{Programas de ayuda computacional}

Los informes sobre tales programas comenzaron a aparecer en la década del 60, a partir de este período, la tecnología se ha desarrollado desde los ordenadores centrales a los microordenadores de multimedia y acceso a la Internet (15).

Las comunidades de aprendizaje han evolucionado del aula tradicional a programas de educación a distancia en línea, en los que los estudiantes se juntan en un ambiente virtual a intercambiar ideas, solucionar problemas, analizar las alternativas y crear los nuevos significados a lo largo de un viaje conectado (16). A continuación se mencionan dos tipos de ayuda computacional:

\section{Aprendizaje con ayuda computacional (CAL)}

Es un método individualizado de autoinstrucción, que ofrece una actividad educativa para los estudiantes (17). Este aprendizaje automatizado puede proporcionar un ambiente rico para programas interactivos bien diseñados que evalúan la integración del
CAL en el plan de estudios. A su vez, ayuda a profesores y a principiantes a apreciar la utilidad de esta metodología en el mundo de la educación en salud (18).

Este tipo de aprendizaje es un encuentro clínico multimedial, centrado en las capacidades de los estudiantes para obtener historias apropiadas, examinaciones físicas, pruebas e impresiones diagnósticas, planes del tratamiento, y evaluaciones razonables de severidad en un paciente virtual simulado. Inicialmente, al estudiante le es presentado un esquema con características del escenario del paciente. Luego, es consultado para ingresar a un set de hipótesis diagnósticas preliminares que podrían ser consideradas, basadas en la información introductoria. Finalmente, cada diagnóstico ingresado es procesado a través de un algoritmo de texto reconocido por el programa (19).

La presentación de respuestas multimediales en el momento apropiado hace a la simulación de caso más interesante al estudiante. Sin embargo, el logro de la calidad del contenido multimedia es uno de los aspectos que más tiempo consume dentro del desarrollo del caso y la evaluación de la entrevista al paciente; los exámenes físicos y las pruebas de laboratorio se individualizan para cada estudiante, a diferencia del caso, del diagnóstico y la terapia que no se individualizan (19).

El sistema descrito compara las acciones emprendidas por el estudiante con las acciones recomendadas por los autores del caso. En esas secciones, el estudiante compara sus respuestas con lo que ha propuesto el autor del caso como la respuesta correcta recomendada (19).

Kaveevivitchai et al. coinciden con Travale, en que a partir de los resultados obtenidos en su estudio el CAL podría sustituir la demostración por parte de los docentes, principalmente frente a la falta de recursos educativos producida por el explosivo aumento de las escuelas de Enfermería a nivel mundial $(17,20)$. 


\section{Instrucción con ayuda computacional (CAI)}

Este sistema consiste en que el usuario del programa selecciona al paciente en una sala de espera virtual y posterior a esto lo atiende, los datos del paciente son únicos para cada caso y consisten en texto, imágenes y los clips de video. Cuando el estudiante ha sugerido un diagnóstico, puede incorporar una sección llamada "más sobre la enfermedad". Ahí puede encontrar información extensa con una gran cantidad de imágenes que incluyen diagnósticos diferenciales y acoplamientos a los sitios de internet clínicos y científicos (15).

Barker explica que un $\mathrm{CD}$ interactivo en educación puede simular situación de vida real y puede incrementar satisfacción en el aprendiz. Además, es posible adquirir una dimensión adicional a través del uso de las funciones interactivas del programa que atraen al usuario activamente, promocionan pensamiento crítico, y pueden ser usados como entretención (21).

Qayumi et al., en un estudio realizado el 2004, demostraron que la inclusión del CAI resulta ser una ganancia inmediata de conocimientos por sobre los métodos tradicionales. Los resultados sugieren que los métodos de aprendizaje computarizados son de mayor ayuda a los estudiantes que no tienen buen rendimiento con los métodos tradicionales (22).

En esta misma línea, al comparar un protocolo experimental del método CAI con la instrucción basada en textos se demostró que el CAI otorga significativamente más conocimientos que el método tradicional sólo con textos y entrega un efecto positivo en el desempeño de estudiantes en su aplicación práctica. En el mismo estudio destaca que habría mayor impacto en aquellos estudiantes con menor rendimiento que en aquéllos con mayor rendimiento (22).

Hudson agrega que la mayor ventaja es que se produce aprendizaje activo, donde los estudiantes prueban sus propios modelos mentales. Esto provoca el desarrollo de estrategias cognoscitivas, más que la adquisición de conocimiento, lo que a la larga fomenta competencias (18).

Así, una clase tutorial interactiva computacional promueve los aspectos de un nivel más alto del proceso de pensamiento como síntesis, análisis y toma de decisiones, provocando que los estudiantes generen su propio pensamiento sobre la trayectoria, la investigación, la interpretación de resultados, la generación de diagnósticos diferenciados y la explicación de las decisiones. Es decir, para practicar el razonamiento en un acercamiento menos complejo que con un paciente verdadero (18).

Por otro lado, Wahlgren et al. plantean que el sistema de aprendizaje se debe basar en casos auténticos, frecuentes e importantes, de modo que cada estudiante pueda obtener experiencia en el diagnostico y manejo. Este autor recalca que estos programas tienen como objetivo apoyar las capacidades de resolución de problemas y las habilidades clínicas de los estudiantes, y proporcionar una base de conocimiento, de manera que apoye un acercamiento a largo plazo de aprendizaje significativo (15).

Aunque no se ha podido demostrar que estos programas mejoran significativamente el rendimiento del estudiante en una examinación escrita tradicional, este mismo autor considera que el programa cobra importancia por varias razones, algunas de ellas son la reutilidad, donde los mismos pacientes auténticos pueden ser utilizados repetidamente, el uso fácil de software estructurado que permite un uso interactivo no en línea, el concepto de aprendizaje sobre enfermedades y su evaluación, que están orientados al paciente, la retroalimentación que se hace con la opinión de un especialista y finalmente la oportunidad de analizar todas las transacciones del estudiante a través de una ficha diaria (15).

Bove menciona algunas desventajas de 
este método, que en ocasiones podría jugar en contra el "analfabetismo digital", la escasa cantidad de personal entrenado y el poco tiempo dedicado a la realización de este procedimiento (23).

\section{Simulación computarizada}

Respecto a ella, se afirma que las experiencias clínicas del instructor con la enseñanza y los pacientes pueden variar enormemente, dependiendo de las designaciones del hospital y las rotaciones clínicas. También, el encuentro clínico en el hospital no presenta siempre el ambiente deseado para los estudiantes. Debido a la importancia de la enseñanza y la variabilidad en experiencias clínicas, hay una necesidad de proporcionar experiencias de aprendizaje equivalentes para todos los estudiantes (24).

De acuerdo a Feingold et al., la simulación clínica está siendo usada para la enseñanza de las destrezas psicomotoras y el desarrollo del pensamiento crítico en estudiantes de la salud. Las razones que se esgrimen para esta tendencia incluyen los cambios de costo en el sistema de salud tradicional, que han resultado en favorecer experiencias de aprendizaje con supervisión, la presencia de pacientes de mayor gravedad e intervenciones de altas tecnologías, lo que requiere de estudiantes mejor preparados y tecnologías de simulación con altos niveles de realismo (25).

Las simulaciones computarizadas pueden ser deseables cuando las situaciones específicas con pacientes no están disponibles o cuando los estudiantes requieren la libertad completa de acción dentro de una situación dada (25). Estas simulaciones permiten el acceso a una variedad amplia de panoramas clínicos, proporcionan la oportunidad para la práctica, y ofrecen una retroalimentación efectiva (24).

Seybert et al. explican que los simuladores humanos de alta fidelidad tienen latidos cardiacos palpables y audibles, ruidos pulmonares y abdominales; parámetros hemodinámi- cos, además de tener la capacidad de hablar en respuesta a las preguntas realizadas. Algunos simuladores pueden ser programados con reacciones fisiológicas apropiadas para un paciente con buen o mal estado clínico (26). El lugar para la simulación está implementado con igual equipamiento de una sala de hospital. Un facilitador entrenado coordina la sesión de simulación desde una sala de control adyacente a través de un vidrio o desde adentro de la misma. Sin embargo, surge la pregunta de si las habilidades adquiridas durante el entrenamiento del simulador se pueden transferir a una situación verdadera y el grado de realismo físico de la simulación varía dependiendo de las capacidades del hardware y del software de un simulador (25).

Lehman et al. explican que hay pocos estudios en este ámbito, y que no proporcionan resultados concluyentes (27).

Halamek et al. sugieren que la habilidad de simular un ambiente clínico requiere atención en los detalles y que las simulaciones más beneficiosas serán aquellas que recrean una situación de vida real (28).

Según Gordon et al. en los videos se incluyen las voces del examinador y del paciente. Por ejemplo, para el nivel sensorial de la punción, los estudiantes ven las manos de los examinadores y oyen su voz, ven el torso de los pacientes y escuchan sus propias voces (29).

Pese a que la confianza percibida no es un indicador exacto del nivel de aprendizaje, Vivekananda-Schmidt et al. explican que es un predictor del éxito del estudiante. Las conclusiones inesperadas de este estudio fueron que menos de la mitad de los estudiantes creyeron que las experiencias clínicas simuladas incrementaron su confianza o mejoraron su competencia clínica y solamente la mitad mencionó que la simulación funciona como un ambiente clínico legítimo. Lo más importante es que el impacto de este recurso tecnológico educativo dependerá probablemente de su calidad (30). 
Seybert A. et al. agregan que la simulación de pacientes ofrece una oportunidad única de aprendizaje y de participación, lo que fomenta el logro de altos niveles de rendimiento clínico (26). Vivekananda-Schmidt et al. concluyen fuertemente que este método favorece la autoconfianza en los estudiantes al ser una tecnología que propicia el aprendizaje en un entorno seguro, mejorando los conocimientos y la capacidad para resolver problemas de tratamiento de los pacientes (30).

Gilbart et al. recalcan la importancia de la retroalimentación dada por el instructor a los estudiantes con respecto a sus desempeños en los contextos de enseñanza, y el tiempo dado a los estudiantes para practicar y hacer preguntas (24). Otros autores han documentado la importancia de retribuir a los alumnos con el uso de estas simulaciones computarizadas.

Marquis et al. estudiaron la utilidad de las simulaciones en la evaluación de los problemas de pacientes como herramienta para continuar la educación de salud y encontraron que proporcionar la retroalimentación con la simulación computarizada era extremadamente importante para los resultados positivos (31).

Un estudio realizado por Gilbart et al. (24), cuyo objetivo era evaluar las habilidades adquiridas por los alumnos en trauma a través de la enseñanza con un simulador, mostró una tendencia hacia la mejora en el grupo de entrenamiento con simulador, pero no alcanzó la significación estadística. Estos resultados indican que el proceso activo de aprender y de practicar de una manera real en el simulador computarizado no generó en el estudiante una manera más abstracta de manejar la situación, en comparación con el uso de protocolos verbales.

Lo anterior es concordante con informes publicados de los modelos computarizados de simulación en la literatura, en la cual estos modelos se han creado para hacer más fácil y más agradable el aprendizaje, realzando así la comprensión y la motivación del estudiante. Bruce destaca el nivel de satisfacción de los estudiantes con la experiencia y con la oportunidad de participar en este tipo de evaluaciones (32). Al igual que en el ECOE, Cioffi destaca que, en este sistema, los estudiantes de más bajas calificaciones valoran más la práctica de destrezas, pues necesitan más oportunidades de ejercitar que los estudiantes con la calificación más alta (33).

Además, Knight et al. llegaron a la conclusión de que una destreza es aprendida mejor en un enfoque sistemático que incluye la práctica de repetición en un ambiente seguro. Junto a ello, mencionan que es poco probable que los ajustes clínicos en el sistema de salud actual puedan suministrar todos los elementos de la práctica requerida por estudiantes (34).

Las experiencias de simulación en un laboratorio nunca reemplazarán las experiencias clínicas con pacientes legítimos, modelos a imitar, y consejeros (34). Sin embargo, según Feingold et al., la contención de costos fuerza a la innovación educativa a que satisfaga la necesidad de proveer experiencias de cuidado con más supervisión (25).

Schiavenato concluye que será necesaria "la reconceptualización de la simulación como herramienta de enseñanza que abarque distintos métodos y una gran cantidad de aplicaciones en todo estudiante de enfermería” (35).

\section{El aprendizaje basado en Web}

La igualdad de acceso a la literatura médica a través de internet ha cambiado la relación profesional-paciente. Ahora los pacientes desafían a los profesionales de la salud, preguntando "por qué se prefiere el tratamiento sugerido A al tratamiento B encontrado en Internet" (36). El autor aclara que el nuevo paradigma de la educación de la salud es la creencia de que los profesionales de la salud son principiantes autodirigidos, con acceso al conocimiento y las habilidades, que eva- 
luarán la información y entregarán cuidados de calidad a sus pacientes. Explica que "el advenimiento de la informática ha proporcionado varios desafíos a la educación de estos profesionales y a la práctica de las ciencias de la salud. Por esta razón, el programa basado en Web fue diseñado con un doble propósito: para proporcionar un expediente comprensivo y longitudinal de las experiencias educativas, y ser herramienta de aprendizaje de gran alcance por el pensamiento crítico que anima y promueve las preguntas clínicas" (36).

También proporciona un conducto para buscar recursos en línea y para formular respuestas a las preguntas presentadas. Del mismo modo intenta consolidar un aprendizaje activo y determinar su impacto potencial en la práctica futura (36).

Kowlowitz et al. explican que en su estudio se diseñó una biblioteca de simulación clínica en línea para enseñar competencias de enfermería geriátrica y centrarse en el cuidado de adultos mayores gravemente enfermos. En este estudio, el realismo del caso, la exactitud de la situación o el problema descrito, y la pertinencia para la práctica fueron calificadas como excelentes o muy buenas por más del $85 \%$ de los usuarios, lo que se debió a la alta calidad del sistema utilizado (37).

$\mathrm{Lu}$ et al. coinciden con Kowlowitz et al., en que el desarrollo de casos es un proceso que requiere tiempo, y el apoyo de una buena tecnología (38). Este último agrega que es imprescindible un equipo de trabajo, que consista en un director de proyecto, de profesores con experiencia en el tema a desarrollar, un consultor educativo, un diseñador web, un editor y un evaluador. Lu et al. destacan que el software y soporte de hardware también son importantes para garantizar una relación fluida entre los usuarios del programa (38).

Otro de los métodos basados en Web, mencionados en la literatura, es el uso de "Wikis". Estos elementos son foros de sitios Web abiertos para que los estudiantes envíen contenido y puedan modificarlo según las necesidades. Dentro del programa se mencionan elementos como prevención de hipotermia para adultos mayores, y un programa de prevención del cáncer de vejiga, basado en el análisis de los datos epidemiológicos (39).

La historia ingresada a Wikis, puede ser seguida en un debate en línea con el fin de favorecer la comunicación y la construcción de conocimientos activamente entre los estudiantes (39).

Se cree que este proceso es crucial en el curso de mantenimiento de la capacidad y la calidad práctica del profesional futuro, puesto que aprenden sobre todo solucionando problemas y haciendo preguntas. Por ello, la última meta es hacer una experiencia de aprendizaje en cada encuentro con pacientes o historias (40).

Fung et al. destacan que la timidez de capacidades es un elemento crítico en la evolución de un principiante autodirigido que intenta identificar necesidades de aprendizaje del futuro, pues pocas iniciativas educativas registran el impacto de este elemento en el proceso de aprendizaje. Este programa proporciona evaluaciones en tiempo real de funcionamiento del programa, reconoce las decisiones de estudiantes individuales, lo cual facilita el desarrollo de prescripciones educativas personalizadas, y proporciona una línea de consecuencia del paso de tiempo en el encuentro con pacientes.

Finalmente, este autor explica que el programa facilita la reforma del plan de estudios con la identificación del objetivo de las necesidades de aprendizaje de los estudiantes y documenta el número y la gama de casos necesarios por estudiante, una característica particularmente útil en la evaluación de programas y acreditación (36).

Por otro lado, Babenko-Mould et al. mencionan que, en gran parte, la investigación respecto a tecnologías basadas en Web en la educación de salud ha involucrado a estudiantes de postgrado. Sin embargo, es también importante percibir cómo influye 
en estudiantes de enfermería de pregrado el aprendizaje mediado por computador (16).

Trasponer esta visión de la educación de salud al ambiente de aprendizaje ayuda a aclarar cómo influye en las interacciones de estudiantes la autoeficacia y la conectividad, y demuestra la necesidad de estudio y de participación en compartir perspectivas sobre las experiencias clínicas (16). Este autor se basa en la Teoría de Bandura de la autoeficacia para apoyar el concepto de que las creencias o confianza percibida de una persona individual para coordinar y llevar una acción específica influyen sobre si ésta es tomada o no.

En relación a esto, Babenko-Mould et al. conceptualizan las diferencias entre las creencias de los estudiantes, la confianza para llevar las capacidades profesionales y participar durante la práctica clínica final (16). Para medir las capacidades profesionales, en su estudio se utilizo el instrumento de enseñanza mediada por computación (SECMLI).

Este instrumento refleja los "conocimientos, habilidades, destrezas, actitudes y el criterio" requeridos en la "entrada de nivel" para practicar "sin peligro y eficazmente". Además, este instrumento mide el grado de confianza que se tiene para llevar a cabo una tarea específica o destreza relacionada con las capacidades profesionales.

Sin considerar el método de enseñanza, los estudiantes demuestran un incremento en sus niveles de confianza en todos los dominios de competencia (16).

"Los estudiantes de enfermería requieren preparación suficiente antes de enfrentarse con pacientes extremadamente enfermos y la compleja tecnología involucrada en el cuidado de esos pacientes. Los modelos de pacientes informatizados pueden empezar a ocupar este rol. Los educadores de enfermería también deben considerar si la tecnología puede abordar la comunicación y la relación interpersonal, el cuidado compasivo y el conocimiento de enfermería" (25).

\section{CONCLUSIONES}

El proceso enseñanza aprendizaje ha tenido grandes cambios desde la década de los 90 en adelante, a consecuencia del avance de la investigación y la tecnología durante el siglo pasado (41). Estas tecnologías, necesarias y altamente deseables, han captado el interés de los estudiantes y los educadores llegando a ocupar un sitial importante al interior de las instituciones formadoras. Si esta tecnología es proyectada a la experiencia clínica, se puede generar mayor seguridad y confianza en los estudiantes previa al contacto con el paciente real, constituyéndose en un gran potencial para la educación en enfermería (42). Sin embargo, el aprendizaje y evaluación en el área de la salud implica múltiples aspectos y es esencial disponer de un instrumento que mida lo que pretende medir. Por ello se debe contar con instrumentos validos y confiables que no sólo consideren el aspecto cognoscitivo sino que permitan al estudiante transferir sus experiencias a un ambiente clínico real, para asegurar que el nuevo graduado posea la capacidad necesaria para solucionar los problemas que plantea el ejercicio profesional.

Si bien la evidencia no mostró una mejora significativa en el desempeño de los estudiantes con ninguna de las cuatro metodologías estudiadas, se pudo observar un mayor desarrollo en la confianza y mejora de la autoeficacia (15).

La experiencia de las autoras en la planificación, elaboración e implementación de algunas de estas metodologías, deja de manifiesto que no son métodos exentos de problemas, especialmente al inicio, por lo que se requiere disponer de un equipo de trabajo dispuesto a liderar los cambios en la docencia, interesado en la capacitación de pares y en desarrollar estas metodologías, incluso a expensas de sus tiempos personales.

Se propone elaborar un proyecto enmar- 
cado en la planificación estratégica de las instituciones educacionales, en el que, idealmente bajo el alero de una oficina de educación en enfermería, se trabaje en el diagnóstico y levantamiento de las necesidades de los estudiantes y de los docentes, para visualizar aquellos cursos en los que estas metodologías pueden ser incorporadas. Asimismo, se debe realizar una capacitación a los docentes para que cuenten con las herramientas necesarias para implementar las metodologías de forma correcta. Este proceso de toma de decisiones debe ser en base a la realidad local, la experiencia del equipo docente y el énfasis curricular del plan de estudio.

Es importante continuar realizando estudios que permitan establecer con más claridad aspectos relevantes del desarrollo de cada metodología para un aprendizaje seguro tanto para el estudiante como para el usuario. Asimismo, es necesario modificar en los docentes el paradigma mental de la enseñanza tradicional que aún persiste por una educación centrada en el estudiante, donde estas metodologías ocupan un papel destacado y acorde con la realidad del estudiante de hoy.

\section{REFERENCIAS}

1. Casas MV. Por qué los niños deben aprender música. Colomb Med [Revista en Internet] 2001; 32(4). Hallado en: http:// www.bioline.org.br/request?rc01038. Acceso el 29 de agosto 2009.

2. Arévalo GA, Maldonado JR. Psicoeducación. Psicocentro [Revista en Internet] 2005. Hallado en: http://www. psicocentro.com/cgi-bin/articulo_s. asp?texto=art51001. Acceso el 10 de septiembre 2009.

3. Barzansky B, Etzel SI. Educational programs in US medical schools, 2002-2003. JAMA. 2003; 290(9): 1190-1196.

4. Toledo JA, Fernández MA, Trejo Mejía
JA, Grijalva MG, Gómez FJ, Ponce ER. Evaluación de la competencia clínica en el posgrado de medicina familiar mediante el Examen Clínico Objetivo Estructurado. Aten Primaria. 2002; 30(7): 435-41.

5. Triviño X, Vásquez A, Mena A, López A, Aldunate M, Varas M, et al. Aplicación del Examen Clínico Objetivo Estructurado (ECOE) en la evaluación final del internado de pediatría en dos escuelas de medicina. Rev Med Chil. 2002; 130(7): 817-24.

6. Diccionario de la lengua española [Internet]. España: Real Academia Española; 2001. Hallado en: http://www.rae.es/rae. html. Acceso el 20 de julio 2009.

7. Parra I. Modelo didáctico para contribuir a la dirección del desarrollo de la competencia didáctica del profesional de la educación en formación inicial [tesis doctoral]. Ciudad de la Habana: Instituto Superior Pedagógico Enrique José Varona; 2002.

8. Ortiz E. Competencias y valores profesionales. Rev Pedagog Univ. 2003; 6(2): 59-64.

9. Jiménez $\mathrm{V}$, Lorenzo $\mathrm{C}$, Rivas $\mathrm{Z}$, Becerra $\mathrm{O}$, Rodríguez O, Rodríguez A. Valoración de un método de evaluación final diferente en la Licenciatura en Enfermería. Rev Cubana Enferm. 1997; 13(2): 86-94.

10. Pedregal M, Molina E, Prados JA, Quesada F, Bonal P, Iglesias C. Evaluación de la competencia clínica de tutores de residentes de medicina familiar y comunitaria. Aten Primaria. 2004; 34(2): 68-72.

11. Harden RM, Stevenson M, Downie WW, Wilson GM. Assessment of clinical competence using objective structured examination. Br Med J. 1975; 22(1): 447-451.

12. Hassell AB. Assesment of specialist registrars in rheumatology: experience of an objective structured clinical examination (OSCE). Rheumatology. 2002; 41(11): 1323-1328.

13. Sloan DA, Plymale MA, Donnelly MB, 
Schwartz RW, Edwards MJ, Bland KI. Enhancing the clinical skills of surgical residents through structured cancer education. Ann Surg. 2004; 239(4): 5616.

14. Kurz JM, Mahoney K, Martin-Plank L, Lidicker J. Objective structured clinical examination and advanced practice nursing students. J Prof Nurs. 2009; 25(3): 186-91.

15. Wahlgren CF, Edelbring S, Fors U, Hindbeck H, Ståhle M. Evaluation of an interactive case simulation system in dermatology and venereology for medical students. BMC Med Educ [Internet] 2006; 6(40). Hallado en: http://www. biomedcentral.com/content/pdf/14726920-6-40.pdf. Acceso el 15 de agosto 2009.

16. Babenko-Mould Y, Andrusyszyn MA, Goldenberg D. Effects of computerbased clinical conferencing on nursing students' self-efficacy. J Nurs Educ. 2004; 43(4): 149-55.

17. Kaveevivitchai C, Chuengkriankrai B, Luecha Y, Thanooruk R, Panijpan B, Ruenwongsa P. Enhancing nursing students' skills in vital signs assessment by using multimedia computer-assisted learning with integrated content of anatomy and physiology. Nurse Educ Today. 2009; 29(1): 65-72.

18. Hudson JN. Computer-aided learning in the real world of medical education: does the quality of interaction with the computer affect student learning? Med Educ. 2004; 38(8): 887-95.

19. Feldman MJ, Barnett GO, Link DA, Coleman MA, Lowe JA, O’Rourke EJ. Evaluation of the Clinical Assessment Project: a computer-based multimedia tool to assess problem-solving ability in medical students. Pediatrics. 2006; 118(4): 1380-7.

20. Travale IL. Computer-assisted instruction for novice nurses in critical care. J Contin Educ Nurs. 2007; 38(3): 132-8.
21. Barker, AM. A case study in instructional design for web-based courses. Nurs Educ Perspect. 2002; 23(4): 183-6.

22. Qayumi AK, Kurihara Y, Imai M, Pachev G, Seo H, Hoshino Y, et al. Comparison of computer-assisted instruction (CAI) versus traditional textbook methods for training in abdominal examination (Japanese experience). Med Educ. 2004; 38(10): 1080-8.

23. Bove, LA. Computer-assisted education for critical care nurses. Crit Care Nurs Clin North Am. 2001; 13(1): 73-81.

24. Gilbart MK, Hutchison CR, Cusimano MD, Regehr G. A computer-based trauma simulator for teaching trauma management skills. Am J Surg. 2000; 179(3): 223-8.

25. Feingold CE, Calaluce M, Kallen MA. Computerized patient model and simulated clinical experiences: evaluation with baccalaureate nursing students. J Nurs Educ. 2004; 43(4): 156-63.

26. Seybert AL, Kobulinsky LR, McKaveney TP. Human patient simulation in a pharmacotherapy course. Am J Pharm Educ [Revista en Internet] 2008; 72(2): 37-44. Hallado en: http://www.ajpe.org/ aj7202/aj720237/aj720237.pdf. Acceso el 8 de septiembre 2009.

27. Lehman K. Clinical nursing instructors' use of handheld computers for student recordkeeping and evaluation. J Nurs Educ. 2003; 42(1): 41-2.

28. Halamek LP, Kaegi DM, Gaba DM, Sowb YA, Smith BC, Smith BE, et al. Time for a new paradigm in pediatric medical education: teaching neonatal resuscitation in a simulated delivery room environment. Pediatrics. 2000 Oct; 106(4): 1-6.

29. Gordon JA, Tancredi DN, Binder WD, Wilkerson WM, Shaffer DW. Assessment of a clinical performance evaluation tool for use in a simulator-based testing environment: a pilot study. Acad Med. 2003;78 (10 Suppl): S45-7. 
30. Vivekananda-Schmidt P, Lewis M, Hassell AB. Cluster randomized controlled trial of the impact of a computer-assisted learning package on the learning of musculoskeletal examination skills by undergraduate medical students. Arthritis Care Res. 2005; 53(5): 764-71.

31. Marquis Y, Chaoulli J, Bordage G, Chabot $J M$, Leclere H. Patient-management problems as a learning tool for the continuing medical education of general practitioners. Med Educ. 1984; 18(2): $117-24$.

32. Bruce SA, Scherer YK, Curran CC, Urschel DM, Erdley S, Ball LS. A collaborative exercise between graduate and undergraduate nursing students using a computer-assisted simulator in a mock cardiac arrest. Nurs Educ Perspect. 2009; 30(1): 22-7.

33. Cioffi, J. Clinical simulations: development and validation. Nurse Educ Today. 2001; 21(6): 477-86.

34. Knight CM. Evaluating a skills centre: the acquisition of psychomotor skills in nursing: a review of the literature. Nurse Educ Today. 1998; 18(6): 441-7.

35. Schiavenato M. Reevaluating simulation in nursing education: beyond the human patient simulator. J Nurs Educ. 2009; 48(7): 388-94.

36. Fung MF, Walker M, Fung KF, Temple L, Lajoie F, Bellemare G et al. An internetbased learning portfolio in resident education: the KOALA multicentre programme. Med Educ. 2000; 34(6): 474-9.

37. Kowlowitz V, Davenport CS, Palmer MH. Development and dissemination of Webbased clinical simulations for continuing geriatric nursing education. J Gerontol Nurs. 2009; 35(4): 37-43.

38. Lu DF, Lin ZC, Li YJ. Effects of a Webbased course on nursing skills and knowledge learning. J Nurs Educ. 2009; 48(2): 70-7.

39. Ciesielka, D. Using a wiki to meet graduate nursing education competencies in collaboration and community health. J Nurs Educ. 2008; 47(10): 473-6.

40. D'Alessandro DM, Lewis TE, D’Alessandro MP. A pediatric digital storytelling system for third year medical students: the virtual pediatric patients. BMC Med Educ [Revista en Internet] 2004; 4(10). Hallado en: http://www.biomedcentral. com/content/pdf/1472-6920-4-10.pdf. Acceso el 15 de agosto 2009.

41. Spoczyk DL. Technology in Education. En Bastable SB, ed. Nurse as Educator: Principles of teaching and learning for nursing practice. Massachusetts: Jones and Bartlett Publishers edition: 2008. p. 515-555.

42. Jeffries PR. Using simulations in nursing education. En: Jeffries PR, ed. Simulations in Nursing Education: From Conceptualization to Evaluation. New York: The National League for Nursing edition; 2007. p. 1-10. 